\title{
Relationship of Malnutrition During Hospitalization With Functional Recovery and Postdischarge Destination in Elderly Stroke Patients
}

\author{
Masaaki Sato, OTR, MS, ${ }^{*}+\neq$ Yoshikazu Ido, OTR, PhD, $\ddagger$ \\ Yasuo Yoshimura, MD, PhD, $\ddagger$ and Hitoshi Mutai, OTR, $\mathrm{PhD}^{*}+$
}

\begin{abstract}
Aim: Malnutrition during hospitalization affects the functional recovery and postdischarge destinations of elderly stroke patients. However, insufficient studies exist about nutritional status during hospitalization in the acute stroke phase in this population. This study determined factors of nutritional status changes during hospitalization in elderly patients in the acute phase of stroke, and investigated the relationship between nutritional status changes and improved activities of daily living and postdischarge destination. Methods: This retrospective observational study included 205 acute-phase stroke patients admitted to Shinshu University Hospital from 2010-2016. Multiple regression analysis was conducted to determine relationships between nutritional status changes, patient characteristics, and improved activities of daily living. Binomial logistic regression analysis was used to determine the relationship between the postdischarge destination and nutritional status changes. Results: Prevalence of malnutrition was $42 \%$ at admission and $76 \%$ at discharge. Factors affecting nutritional status changes at admission included Geriatric Nutritional Risk Index $(\beta=-0.35, P<.001)$ and Barthel Index $/$ feeding $(\beta=0.22$, $P=.002)$, and factors during hospitalization included age $(\beta=-0.21, P<.001)$, ischemic stroke $(\beta=0.16, P=.008)$, and National Institute of Health Stroke Scale score $(\beta=-0.29, P<.001)$. Significant associations of changes in geriatric nutritional risk index occurred with improved activities of daily living during hospitalization $(\beta=0.26, P<.001)$ and discharge to home (odds ratio $=1.11,95 \%$ confidence interval: $1.03-1.19, P=.008)$. Conclusion: Many elderly patients in the acute phase of stroke with malnutrition exhibited worsening conditions during hospitalization, which was negatively associated with their activities of daily living abilities and postdischarge destinations. Nutritional status changes and associated factors must be evaluated during hospitalization in this patient population.
\end{abstract}

Key Words: Stroke-malnutrition--acute phase-elderly-geriatric nutritional risk index

(C) 2019 Elsevier Inc. All rights reserved.

\footnotetext{
From the *Shinshu University, Graduate School of Medicine, Department of Medical Sciences, Matsumoto, Nagano, Japan; +School of Health Sciences, Shinshu University School of Medicine, Matsumoto, Nagano, Japan; and ‡Rehabilitation Center, Shinshu University Hospital, Matsumoto, Nagano, Japan.

Received December 28, 2018; revision received March 9, 2019; accepted April 9, 2019.

Grant support: University Grants in Shinshu University.

Address correspondence to Hitoshi Mutai OTR, PhD, School of Health Sciences, Shinshu University School of Medicine, 3-1-1 Asahi, Matsumoto, Nagano 390-8621, Japan. E-mail address:

hitmutai@shinshu-u.ac.jp.

1052-3057/\$ - see front matter

(c) 2019 Elsevier Inc. All rights reserved.

https://doi.org/10.1016/j.jstrokecerebrovasdis.2019.04.012
}

\section{Introduction}

Reports of the incidence of malnutrition among stroke patients during the acute phase vary widely, from $6.1 \%$ to $79 \%,{ }^{1,2}$ and malnutrition during patients' hospital stay reportedly interferes with improvements in activities of daily living (ADL) ${ }^{3,4}$ and negatively affects the postdischarge destination. ${ }^{5,6}$

Several currently available nutritional status assessment tools are applicable to stroke patients; including various biochemical and physical measurement markers, the Subjective Global Assessment, ${ }^{7}$ and the Mini Nutritional Assessment. ${ }^{8}$ Despite these options, and although advanced age has been identified as a risk factor for both 
malnutrition and stroke, no standard tool exists for the assessment of this complication in elderly stroke patients. However, the geriatric nutritional risk index (GNRI), ${ }^{9}$ a tool used to assess the nutritional statuses of elderly individuals, has been recognized as a potentially effective tool for nutritional status assessments in elderly stroke patients ${ }^{3,4}$ and for those with communication difficulties consequent to consciousness disorders or dementia. ${ }^{3}$ The GNRI is also appropriate for acute-phase stroke patients, many of whom experience consciousness disorders or dementia. However, few studies have used the GNRI to assess the nutritional statuses of elderly acute-phase stroke patients. ${ }^{3}$ Furthermore, no study has conducted a multivariate analysis to examine factors affecting changes in malnutrition among elderly acute-phase stroke patients during hospital stay or postdischarge, despite the importance of these factors in the functional recovery of elderly acute-phase stroke patients.

Several studies ${ }^{3,10}$ have evaluated the effects of nutritional status during hospital stay on improvements in ADL among stroke patients in general and specifically on the outcome of discharge to home. These reports suggest that an association exists between malnutrition at hospital admission and malnutrition at discharge. They also indicated that acute-phase stroke patients experience worsening of malnutrition during the hospital stay. ${ }^{5,11}$ Although the nutritional status at admission is important, a worsening nutritional status during hospitalization would have a much greater effect on ADL and the possibility of being discharged to home.

We aimed to survey the nutritional statuses of elderly acute-phase stroke patients during their hospital stays and to investigate the factors affecting changes in the patients' nutritional statuses during hospitalization and at discharge. We also investigated the relationships between changes in the nutritional statuses of elderly acute-phase stroke patients with improvements in ADL and the possibility of being discharged to home.

\section{Materials and Methods}

\section{Design and Setting}

This retrospective observational study was conducted at Shinshu University Hospital, which includes an Advanced Emergency Medical Care Center, in accordance with the STROBE statement. ${ }^{12}$ At this hospital, stroke patients are admitted to and provided with acute medical care at the Stroke Care Unit, Department of Neurosurgery, and the Neurology Ward. Rehabilitation with fullrisk management and control is provided to patients as early as possible.

\section{Participants}

Overall, 410 stroke patients aged greater than or equal to 65 years were admitted to Shinshu University Hospital between April 1, 2010 and September 30, 2016. Diagnoses of stroke were made by a physician based on clinical symptoms, as well as computed tomography, magnetic resonance imaging, and other diagnostic imaging, in accordance with the ICD-10 classification. After applying the exclusion criteria and excluding cases with missing data, 205 patients were included in the sample population (Fig 1).

\section{Characteristics of the Participants}

All data were retrieved from the Shinshu University Hospital electronic medical records, including the following background characteristics: age, sex, comorbidities, length of hospital stay (days), destination (home, transfer), height, weight, body mass index (BMI), use of long-

Patients admitted for acute care (aged $\geq 65$ years)

$$
n=410
$$

(April 1, 2010 - September 30, 2016)

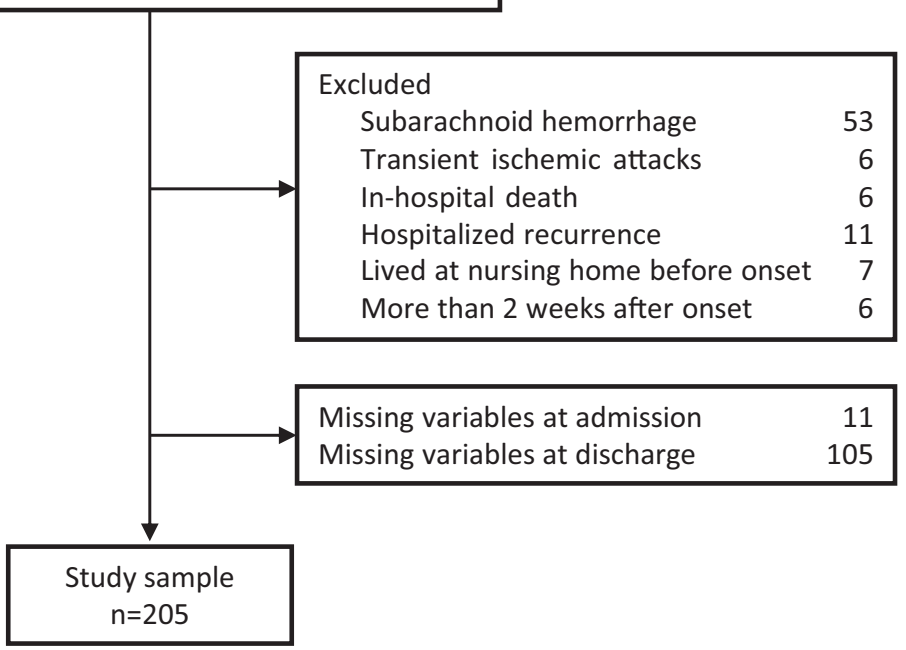

Figure 1. Flow diagram of the study. 
term care insurance prior to hospitalization (yes/no), premorbid ADL dependency (modified Rankin scale [mRS] $\geq 3$ ), cohabitation (yes/no), type of stroke (ischemic, hemorrhagic), and focal hemisphere (right, left, bilateral). Comorbidities were assessed using the Charlson Comorbidity Index (CCI). ${ }^{13}$ The severity of stroke during hospitalization was assessed using the NIHSS, ${ }^{14}$ which was previously used as a standard assessment tool in stroke patients. The severities of dysphagia at admission and discharge were assessed using the Food Intake LEVEL Scale (FILS). ${ }^{15}$ Levels 1-3, 4-6, 7-10, and level 10 indicate no oral intake, oral intake and alternate nutrition, oral intake alone (but medical considerations are given), and normal intake, respectively.

\section{Nutritional Assessment}

Nutritional status assessment was based on GNRI at admission and discharge. This tool is based on a calculation that utilizes three variables: serum albumin (Alb), height, and body weight.

The following formula was utilized:

$(1.489 \times \mathrm{alb}[\mathrm{g} / \mathrm{L}])$

$+(41.7 \times$ actual body weight $[\mathrm{kg}] /$ ideal body weight $[\mathrm{kg}])$.

The ideal body weight was calculated using the patient's height and a BMI $\left(\mathrm{kg} / \mathrm{m}^{2}\right)$ of 22 , as reported by previous studies conducted in Japan and overseas. ${ }^{16}$ The results of the GNRI assessment were categorized as follows: GNRI greater than 98, absent; 92 to less than or equal to 98 , low; 82 to less than 92, moderate; and less than 82, major. ${ }^{9}$ Change in the nutritional status during hospitalization (ie, change in GNRI value) was calculated by subtracting the GNRI at admission from the GNRI at discharge.

\section{Evaluation of Improvement in ADL and Destination}

During hospitalization, the Barthel Index (BI), ${ }^{17}$ which has been applied previously to stroke patients, was used to assess ADL within 3 days of hospital admission and discharge. Two indices were used to assess improvement in ADL during the hospital stay: The BI at discharge and the percent change in $\mathrm{BI}$ were used to indicate the ADL level at discharge and changes in ADL level during hospitalization, respectively. Increases and decreases were calculated as (discharge BI - admission BI)/(100 admission BI) and (discharge BI - admission BI)/(admission $\mathrm{BI})$, respectively. ${ }^{18}$

Two postdischarge destination categories, discharge to home or transfer to another hospital group, for the purpose of continued rehabilitation, were also applied.

\section{Statistical Analysis}

Differences in characteristics between the 205 patients included in the analysis and the 116 patients with missing data were determined using chi-square and Mann-Whitney $U$ tests. Within the analytical population, comparisons of data at admission and discharge were conducted using the paired $t$ test and Wilcoxon test. Comparisons of patient attributes between the discharge to home and transfer at discharge groups were conducted using the chi-square test and Mann-Whitney $U$ test.

Factors with potential effects on changes in nutritional status during hospitalization, as indicated by the GNRI at discharge as well as the degree of change in the GNRI, were determined using a stepwise multivariate analysis. Highly correlated variables were selected based on previous research findings ${ }^{19}$ and the authors' clinical empirical knowledge.

Multivariate stepwise regression analysis of BI at discharge and percent change in BI was performed to determine the association of changes in nutritional status with improvement in ADL and the possibility of discharge to home. A binomial logistic regression analysis was also performed using elimination methods via the likelihood ratio with the postdischarge destination (home or transfer). The predictive variables were GNRI at admission or amount of change in GNRI, age, ${ }^{17,20}$ sex, ${ }^{17,20} \mathrm{CCI}^{20}$ stroke type (ischemic, hemorrhagic), ${ }^{17,20,21}$ focal hemisphere (right, left, bilateral), ${ }^{17,20}$ premorbid mRS (mRS $<3$ or $\geq 3),{ }^{17}$ cohabitation (yes/no), ${ }^{21}$ NIHSS at admission, ${ }^{17,20}$ BI at admission, ${ }^{17,20,21}$ and FILS at admission. ${ }^{22}$

$\mathrm{G}$ *Power3.1 was used to determine the sample size for multivariate analysis. ${ }^{23}$ This study met the required number of cases $(n=123)$. For all statistical analyses, statistical standard of significance was set at less than $5 \%$. The analyses were performed using IBM SPSS Statistics version 25.0 (IBM Corp., Armonk, NY).

\section{Ethics}

This study was conducted in accordance with the Declaration of Helsinki ${ }^{24}$ and ethical guidelines for medical and health research involving human subjects. ${ }^{25}$ This study was approved by the Ethics Committee of Shinshu University (approval number 3556). As this was a retrospective study, the Shinshu University Hospital internet homepage published information about the study to allow patients the opportunity to withdraw from participation.

\section{Results}

Basic attributes of the 205 study participants included in the analysis and the 116 patients with missing data are shown in Table 1. These groups differed significantly in terms of the hospital stay length (days), total CCI score, stroke subtype, cohabitation, BI at admission, and postdischarge destination. The 205 analyzed participants had a mean age of $77 \pm 7$ years and a median GNRI value at admission of 100 . 
Table 1. Baseline characteristics of study sample and individuals excluded for missing data

\begin{tabular}{|c|c|c|c|c|}
\hline Characteristic & & $\begin{array}{l}\text { Included participants } \\
(\mathrm{n}=205)\end{array}$ & $\begin{array}{l}\text { Excluded participants } \\
(\mathrm{n}=116)\end{array}$ & $P$ value \\
\hline Age & Mean \pm SD & $77 \pm 7$ & $78 \pm 8$ & .268 \\
\hline Sex & Male/Female & $114 / 91$ & $67 / 49$ & .709 \\
\hline Length of stay & Mean \pm SD & $23 \pm 17$ & $15 \pm 11$ & $<.001$ \\
\hline $\mathrm{CCI}$ & Median (IQR) & $2(1-3)$ & $1(0-2)$ & .027 \\
\hline Onset & First/recurrent (\%) & $150(73) / 55(27)$ & $83(72) / 33(28)$ & .755 \\
\hline \multirow[t]{6}{*}{ Stroke subtype } & $(\%)$ & & & \\
\hline & Lacunar infarction & $27(13)$ & $30(26)$ & .004 \\
\hline & Atherothrombotic infarction & $57(28)$ & $29(25)$ & .586 \\
\hline & Cardioembolic infarction & $45(22)$ & $18(15)$ & .163 \\
\hline & Other types of infarction & $20(10)$ & $14(12)$ & .518 \\
\hline & Intracerebral hemorrhage & $56(27)$ & $25(22)$ & .253 \\
\hline Lesion & Right/left/both (\%) & $103(50) / 94(46) / 8(4)$ & $55(47) / 59(51) / 2(2)$ & .671 \\
\hline Living situation & Alone/with family (\%) & $20(10) / 185(90)$ & $22(19) / 94(81)$ & .019 \\
\hline $\begin{array}{l}\text { Premorbid dependence } \\
\quad(\text { premorbid } \mathrm{mRS} \geq 3 \text { ) }\end{array}$ & Yes $(\%)$ & $32(16)$ & $19(16)$ & .871 \\
\hline NIHSS at admission & Median (IQR) & $6(2-19)$ & $5(2-10)$ & .068 \\
\hline GNRI at admission & Median (IQR) & $100(93-106)$ & $100(95-104)$ & .964 \\
\hline FILS at admission & Median (IQR) & $2(2-9)$ & $7(1-9)$ & .896 \\
\hline $\mathrm{BI}$ at admission & Median (IQR) & $5(0-45)$ & $17.5(0-65)$ & .008 \\
\hline Discharge home & Yes $(\%)$ & $54(26)$ & $48(41)$ & .005 \\
\hline
\end{tabular}

BI, Barthel index; CCI, Charlson comorbidity index; FILS, food intake level scale; GNRI, geriatric nutritional risk index; IQR, interquartile range; mRS, modified Rankin scale; NIHSS, National Institute of Health Stroke Scale; SD, standard deviation.

Table 2 presents comparisons of all indices related to nutrition. Notably, both the FILS and BI had improved significantly at discharge relative to the values at admission. However, BMI, Alb, and GNRI exhibited significant decreases at discharge relative to admission. The results of multivariate stepwise regression analysis of potential factors affecting GNRI during hospitalization are shown in Table 3. Here, age $(\beta=-0.16, P<.001)$, ischemic stroke $(\beta=0.12$, $P=.008)$, NIHSS at admission $(\beta=-0.22, P<.001)$, GNRI at admission $(\beta=0.64, P<.001)$, and $\mathrm{BI}$ feeding at admission ( $\beta=0.16, P=.002)$ were all found to be associated with the GNRI at discharge. All of these factors were also found to affect changes in the GNRI (age: $\beta=-0.21, P<.001$; ischemic stroke: $\beta=0.16, P=.008$; NIHSS at admission: $\beta=$ $-0.29, P<.001$; GNRI at admission: $\beta=-0.35, P<.001$; BI feeding at admission: $\beta=0.22, P=.002$ ).

Table 4 presents factors affecting improvement in ADL during hospitalization according to the $\mathrm{BI}$ at discharge

Table 2. Nutritional parameters, swallowing function, and activities of daily living at admission and at discharge in 205 patients with stroke

\begin{tabular}{|c|c|c|c|c|}
\hline Characteristic & $\mathrm{n}=205$ & Admission & Discharge & $P$ value \\
\hline BMI & Mean \pm SD & $22.5 \pm 3.2$ & $21.7 \pm 3.3$ & $<.001 *$ \\
\hline Alb & Median (IQR) & $3.9(3.6-4.2)$ & $3.3(2.9-3.7)$ & $<.001^{\dagger}$ \\
\hline GNRI & Median (IQR) & $101.0(93.7-106.7)$ & $89.5(81.2-97.8)$ & $<.001 *$ \\
\hline Nutrition-related risk & Number $(\%)$ & & & \\
\hline Absent $($ GNRI > 98) & & $119(58)$ & $50(24)$ & $<.001^{\ddagger}$ \\
\hline Low $(98 \geq$ GNRI $\geq 92)$ & & $43(21)$ & $36(18)$ & \\
\hline Moderate $(92>$ GNRI $\geq 82)$ & & $31(15)$ & $66(32)$ & \\
\hline Major $($ GNRI < 82) & & $12(6)$ & $53(26)$ & \\
\hline FILS & Median (IQR) & $2(2-9)$ & $7(3-9)$ & $<.001^{\dagger}$ \\
\hline BI & Median (IQR) & & & \\
\hline Total & & $5(0-45)$ & $45(5-85)$ & $<.001^{\dagger}$ \\
\hline Feeding & & $0(0-10)$ & $5(0-10)$ & $<.001^{\dagger}$ \\
\hline
\end{tabular}

Alb, serum albumin; BI, Barthel index; BMI, body mass index; FILS, food intake level scale; GNRI, geriatric nutritional risk index; IQR, interquartile range; $\mathrm{SD}$, standard deviation.

* paired $t$ test.

${ }^{+}$matched pair test of Wilcoxon.

$\ddagger_{\text {chi-square test. }}$ 
Table 3. Multivariate stepwise regression analysis for GNRI during hospitalization

\begin{tabular}{lcrrr}
\hline & \multicolumn{2}{c}{ GNRI at discharge $(\mathrm{n}=205)$} & \multicolumn{2}{c}{ GNRI change $(\mathrm{n}=205)$} \\
\cline { 2 - 4 } Variable & $\beta$ coefficient & $P$ value & & $\beta$ coefficient \\
\hline Age & -0.16 & $<.001$ & -0.21 & $<$ value \\
Stroke type: Ischemic & 0.12 & .008 & 0.16 & .008 \\
NIHSS at admission & -0.22 & $<.001$ & -0.29 & $<.001$ \\
GNRI at admission & 0.64 & $<.001$ & -0.35 & .001 \\
BI feeding at admission & 0.16 & .002 & 0.22 & 0.412 \\
Adjusted R & 0.669 & & 002 \\
\hline
\end{tabular}

BI, Barthel index; GNRI, geriatric nutritional risk index; GNRI change, GNRI at discharge-GNRI at admission; NIHSS, National Institute of Health Stroke Scale.

Table 4. Multivariate stepwise regression analysis of improvements in activities of daily living

\begin{tabular}{|c|c|c|c|c|}
\hline \multirow[b]{2}{*}{ Variable } & \multicolumn{2}{|c|}{ BI at discharge $(n=205)$} & \multicolumn{2}{|c|}{ BI change* $(n=202)$} \\
\hline & $\beta$ coefficient & $P$ value & $\beta$ coefficient & $P$ value \\
\hline Premorbid dependence $(\mathrm{mRS} \geq 3)$ & -0.16 & $<.001$ & -0.20 & $<.001$ \\
\hline Stroke type: Ischemic & 0.10 & .029 & & NS \\
\hline Lesion: right & & NS & -0.12 & .048 \\
\hline NIHSS at admission & -0.29 & $<.001$ & -0.25 & .001 \\
\hline $\mathrm{BI}$ at admission & 0.43 & $<.001$ & 0.17 & .019 \\
\hline GNRI change & 0.19 & $<.001$ & 0.26 & $<.001$ \\
\hline Adjusted $\mathrm{R}^{2}$ & 0.637 & & 0.358 & \\
\hline
\end{tabular}

BI: Barthel index; BI change, (discharge BI - admission BI)/(100 - admission BI) or (discharge BI - admission BI)/(admission BI); GNRI, geriatric nutritional risk index; GNRI change, GNRI at discharge-GNRI at admission; mRS, modified Rankin scale; NIHSS, National Institute of Health Stroke Scale; NS, not significant.

*BI change $(n=202): 3$ patients with a BI at admission $=100$ excluded from the analysis.

$(\mathrm{n}=205)$. This analysis identified the level of premorbid dependency $(\beta=-0.16, P<.001)$, ischemic stroke $(\beta=0.10$, $P=.029)$, NIHSS at admission $(\beta=-0.29 P<.001)$, BI at admission $(\beta=0.43, P<.001)$, and degree of change in the GNRI $(\beta=0.19, P<.001)$ as factors affecting the total BI score at discharge. Premorbid dependence $(\beta=-0.20, P<.001)$, NIHSS at admission $(\beta=-0.25, P=.001)$, BI at admission $(\beta=0.17, P=.019)$, and the amount of change in GNRI $(\beta=0.26, P<.001)$ were also identified as factors affecting the percent change in $\mathrm{BI}$ during hospitalization, together with a right hemispheric lesion $(\beta=-0.12, P=.048)$.

Table 5. Binomial logistic regression analysis for home discharge

\begin{tabular}{lll}
\hline Variable & $\begin{array}{l}\text { Home discharge } \\
\text { OR }(95 \% \text { CI })\end{array}$ & $P$ value \\
\hline NIHSS at admission & $0.82(0.70-0.96)$ & .012 \\
BI at admission & $1.05(1.03-1.07)$ & $<.001$ \\
GNRI change & $1.11(1.03-1.19)$ & .008 \\
\hline
\end{tabular}

BI, Barthel index; CI, confidence interval; GNRI, geriatric nutritional risk index; GNRI change, GNRI at discharge-GNRI at admission; NIHSS, National Institute of Health Stroke Scale; OR, odds ratio.

Hosmer-Lemeshow test: $P=.874$.
Fifty-four (26\%) and 151 (74\%) of the 205 analyzed patients were discharged to home or transferred, respectively. Accordingly, a binomial logistic regression analysis using the elimination methods via the likelihood ratio with the postdischarge destination (home or transfer) was also performed. This analysis identified the NIHSS at admission (odds ratio [OR]: 0.82, 95\% confidence interval [CI] 0.70-0.96, $P=.012$ ), BI at admission (OR: 1.05, 95\% CI $1.03-1.07, P<.001)$, and the degree of change in GNRI (OR: $1.11,95 \%$ CI $1.03-1.19, P=.008)$ in relation to the postdischarge destination (Table 5).

\section{Discussion}

Factors Related to the Nutritional Status and Change in Nutritional Status During Hospital Stay

In this comparison of the nutritional statuses of elderly acute-phase stroke patients at admission and discharge, we found that all three investigated nutritional parameters (BMI, Alb, and GNRI) were lower at discharge than at admission, indicating a worsening nutritional status. We further identified age, stroke subtype, NIHSS at admission, and BI feeding as factors influencing the nutritional status at discharge and the degree of change in this parameter during hospitalization. Notably, the GNRI at admission had a negative effect on changes in the 
nutritional status but had a positive effect on the nutritional status at discharge.

Consistent with our findings, age has been reported as a risk factor for malnutrition among hospitalized ${ }^{26,27}$ and acute stroke patients. However, hemorrhagic stroke is thought more likely than ischemic stroke to lead to malnutrition. ${ }^{28}$ As the present study did not find a difference in nutritional status at admission between patients with ischemic and hemorrhagic stroke, the latter may have required higher amounts of nutrition than the former during the course of brain function recovery, as hemorrhagic stroke is generally associated with more severe disease. ${ }^{29}$ In other words, the brain damage associated with hemorrhagic stroke occurs over a wider region, and therefore additional nutrition is required to recover brain function. However, the relationship between brain lesion type and nutritional status remains insufficiently investigated and requires further study.

A low level of independence with eating behaviors at admission is related to nutritional status. Eating-related disorders are risk factors for malnutrition, ${ }^{30}$ and oral intake of food rather than intravenous feeding is essential to ensuring a nutritional balance. Previously, the nutritional status at admission was found to affect the nutritional status at discharge, ${ }^{31}$ and, consistent with that report, we found that a good nutritional status at admission persisted at discharge. In contrast, patients with a good nutritional status at admission exhibited a worse nutritional status during their hospital stay. Accordingly, careful attention should be given to the nutritional management of hospitalized patients with a good nutritional status at admission.

A previous meta-analysis of several studies reported that a patient's characteristics during hospitalization could be used to predict the long-term nutritional status. ${ }^{19}$ Accordingly, factors identified in this study as affecting the nutritional status during hospitalization and at discharge provide novel and clinically beneficial prognostic predictors for the evaluation of patients hospitalized during the acute phase of stroke.

\section{Relationship Between Improvement in ADL and Nutritional Status During Hospital Stay}

In our analysis of potential factors associated with improvements in ADL, we identified the degree of change in the GNRI during hospitalization as an independent factor; the GNRI at admission showed no association. A study of hospitalized patients in the acute phase of stroke, found that energy intake during the first week after admission affected improvement in ADL. ${ }^{32}$ This previous study finding supports our results, suggesting the importance of focusing more attention on the nutritional status of acute-phase stroke patients during hospitalization. This is because deterioration in the nutritional status at this phase can affect improvements in ADL even with a good nutritional status in patients at admission.
Relationship Between the Postdischarge Destination and Nutritional Status During the Hospital Stay

Although the GNRI at admission was not associated significantly with the likelihood of discharge to home, we found that the degree of change in the GNRI during hospitalization was an independent factor influencing clinical decisions of the postdischarge destination. A previous study reported that nutrition-related disorders negatively affected the ability of convalescent stroke patients admitted to rehabilitation hospitals to be discharged to home. ${ }^{6}$ However, no previous report addressed whether changes in the nutritional status during hospitalization was associated with the postdischarge destinations of elderly acute-phase stroke patients. It is possible that sufficient in-hospital management of nutritional status could help prevent a worsening status and thus promote discharge to home among elderly patients in the acute phase of stroke.

In this study, we used the GNRI to investigate the nutritional status of acute-phase stroke patients aged greater than or equal to 65 years during hospitalization. Notably, $41 \%$ and $76 \%$ of these patients exhibited malnutrition at admission and discharge, respectively. We identified age, stroke type, NIHSS at admission, GNRI at admission, and BI feeding at admission as factors related to a worsening of the nutritional status during hospitalization. In other words, patients who are of advanced age with cerebral hemorrhage, severe stroke, good nutritional status at admission, and difficulty with independent feeding face a higher risk of a worsening nutritional status. These results underscore the importance of careful nutritional status management as early as possible. Moreover, the degree of change in GNRI was identified as an independent factor associated with improvement in ADL and discharge to home, suggesting that the outcomes of elderly stroke patients would benefit from careful attention to changes in the nutritional status during the acute phase.

\section{Limitations}

The present study had some limitations of note. For example, many subjects had missing data at discharge. These patients tended to have shorter hospital stays, lower complication indices, a high proportion of lacunar infarction, and higher BI at admission; they were also more likely to live alone and be discharged to home. In other words, patients with missing data exhibited higher levels of ADL independence and more stable symptoms, which enabled them to be discharged to home earlier and at greater frequency. Additionally, many of these patients were determined not to require nutritional status assessments at discharge. Therefore, the present study may have excluded patients who tended to have less severe symptoms. This may have resulted in selection bias, and thus the results may not be generalizable to all patients with stroke. Moreover, the endpoint of the present study, the destination after discharge from an acute care hospital, predicted the 
outcome of a short-term hospital stay. Therefore, further studies are needed to investigate the effect of changes in the nutritional status during the acute phase on long-term outcomes. Finally, although the GNRI was measured at both admission and discharge, the follow-up periods were not uniform and therefore we could not identify the point at which the GNRI value started to decline. This parameter will require investigation in the future.

\section{Conflicting Interests}

The authors declare no conflict of interest.

\section{References}

1. Foley NC, Salter KL, Robertson J, et al. Which reported estimate of the prevalence of malnutrition after stroke is valid. Stroke 2009;40:E66-E74.

2. Hafsteinsdottir TB, Mosselman M, Schoneveld C, et al. Malnutrition in hospitalised neurological patients approximately doubles in 10 days of hospitalisation. J Clin Nurs 2010;19:639-648.

3. Kokura Y, Maeda K, Wakabayashi H, et al. High nutritional-related risk on admission predicts less improvement of functional independence measure in geriatric stroke patients: a retrospective cohort study. J Stroke Cerebrovasc Dis 2016;25:1335-1341.

4. Nii M, Maeda K, Wakabayashi H, et al. Nutritional improvement and energy intake are associated with functional recovery in patients after cerebrovascular disorders. J Stroke Cerebrovasc Dis 2016;25:57-62.

5. Gariballa SE, Parker SG, Taub N, et al. Influence of nutritional status on clinical outcome after acute stroke. Am J Clin Nutr 1998;68:275-281.

6. Nishioka S, Wakabayashi H, Yoshida T. Accuracy of nonparalytic anthropometric data for nutritional screening in older patients with stroke and hemiplegia. Eur J Clin Nutr 2017;71:173-179.

7. Poulia KA, Yannakoulia M, Karageorgou D, et al. Evaluation of the efficacy of six nutritional screening tools to predict malnutrition in the elderly. Clin Nutr 2012;31:378-385.

8. Guigoz Y. The mini nutritional assessment (MNA (R)) review of the literature - What does it tell us? J Nutr Health Aging 2006;10:466-485.

9. Bouillanne O, Morineau G, Dupont C, et al. Geriatric nutritional risk index: a new index for evaluating at-risk elderly medical patients. Am J Clin Nutr 2005;82:777-783.

10. Shen HC, Chen HF, Peng LN, et al. Impact of nutritional status on long-term functional outcomes of post-acute stroke patients in Taiwan. Arch Gerontol Geriatr 2011;53:e149-e152.

11. Yoo SH, Kim JS, Kwon SU, et al. Undernutrition as a predictor of poor clinical outcomes in acute ischemic stroke patients. Arch Neurol 2008;65:39-43.

12. Von Elm E, Altman DG, Egger M, et al. The strengthening the reporting of observational studies in epidemiology (STROBE) statement: guidelines for reporting observational studies. Lancet 2007;370:1453-1457.

13. Charlson ME, Pompei $\mathrm{P}$, Ales KL, et al. A new method of classifying prognostic comorbidity in longitudinal studies: development and validation. J Chronic Dis 1987;40:373-383.

14. Kasner SE. Clinical interpretation and use of stroke scales. Lancet Neurol 2006;5:603-612.
15. Kunieda K, Ohno T, Fujishima I, et al. Reliability and validity of a tool to measure the severity of dysphagia: the food intake level scale. J Pain Symptom Manage 2013;46:201-206.

16. Yamada K, Furuya R, Takita T, et al. Simplified nutritional screening tools for patients on maintenance hemodialysis. Am J Clin Nutr 2008;87:106-113.

17. Veerbeek JM, Kwakkel G, Van Wegen EEH, et al. Early prediction of outcome of activities of daily living after stroke a systematic review. Stroke 2011;42:1482-1488.

18. Kugler C, Altenhoner $\mathrm{T}$, Lochner $\mathrm{P}$, et al. Does age influence early recovery from ischemic stroke? A study from the Hessian Stroke Data Bank. J Neurol 2003;250:676-681.

19. Chen N, Li Y, Fang J, et al. Risk factors for malnutrition in stroke patients: a meta-analysis. Clin Nutr 2017; S0261-5614:31434-31436.

20. Mees M, Klein J, Yperzeele L, et al. Predicting post-discharge destination after stroke: a systematic review. Clin Neurol Neurosurg 2016;142:15-21.

21. Itaya T, Murakami Y, Ota A, et al. Assessment model to identify patients with stroke with a high possibility of discharge to home a retrospective cohort study. Stroke 2017;48:2812-2818.

22. Pinedo S, Erazo P, Tejada P, et al. Rehabilitation efficiency and destination on discharge after stroke. Eur J Phys Rehabil Med 2014;50:323-333.

23. Faul F, Erdfelder E, Buchner A, et al. Statistical power analyses using $\mathrm{G}^{*}$ Power 3.1: tests for correlation and regression analyses. Behav Res Methods 2009;41:1149-1160.

24. World Medical Association. World Medical Association Declaration of Helsinki ethical principles for medical research involving human subjects. JAMA 2013;310:2191-2194.

25. Ministry of Health, Labor and Welfare, Japan. Ethical Guidelines for Medical and Health Research Involving Human Subjects. https://www.mhlw.go.jp/file/06-Seisakujouhou-10600000-Daijinkanboukouseikagakuka/ 0000080278.pdf (2015, Accessed November 21, 2018).

26. Incalzi RA, Landi F, Cipriani L, et al. Nutritional assessment: a primary component of multidimensional geriatric assessment in the acute care setting. J Am Geriatr Soc 1996;44:166-174.

27. Wakabayashi H, Sakuma K. Rehabilitation nutrition for sarcopenia with disability: a combination of both rehabilitation and nutrition care management. J Cachexia Sarcopenia Muscle 2014;5:269-277.

28. Choi-Kwon S, Yang YH, Kim EK, et al. Nutritional status in acute stroke: undernutrition versus overnutrition in different stroke subtypes. Acta Neurol Scand 1998;98:187-192.

29. Andersen KK, Olsen TS, Dehlendorff C, et al. Hemorrhagic and ischemic strokes compared stroke severity, mortality, and risk factors. Stroke 2009;40:2068-2072.

30. Jonsson AC, Lindgren I, Norrving B, et al. Weight loss after stroke - A population-based study from the Lund stroke register. Stroke 2008;39:918-923.

31. Dennis M, Collaboration FT. Poor nutritional status on admission predicts poor outcomes after stroke Observational data from the Food trial. Stroke 2003;34: 1450-1455.

32. Kokura Y, Wakabayashi H, Nishioka S, et al. Nutritional intake is associated with activities of daily living and complications in older inpatients with stroke. Geriatr Gerontol Int 2018;18:1334-1339. 